\title{
High-speed kymography identifies the immediateeffects of voiced vibration in healthy vocal folds
}

\author{
Regina Aparecida Pimenta', María Eugenia Dájer², Adriana Hachiya3 ${ }^{3}$, Gislaine Ferro Cordeiro ${ }^{4}$, Domingos Hiroshi Tsuji ${ }^{5}$, \\ Arlindo Neto Montagnoli 6 .
}

1) Master in Bio-Engineering. Doctorate. Post Graduation in Bio-Engineering - University of São Paulo - São Carlos.
2) Doctor in Sciences. Post-Doctorate. Department of Otolaryngology, School of Medicine, University of São Paulo.
3) Doctor in Sciences. Department of Otolaryngology, School of Medicine, University of São Paulo.
4) Master in Sciences of Otolaryngology. Doctorate. Department of Otolaryngology, School of Medicine, University of São Paulo.
5) Professor in the Department of Otolaryngology, School of Medicine, University of São Paulo.
6) Doctor in Sciences. Professor in the Department of Electric Engineering of University Federal of São Carlos.

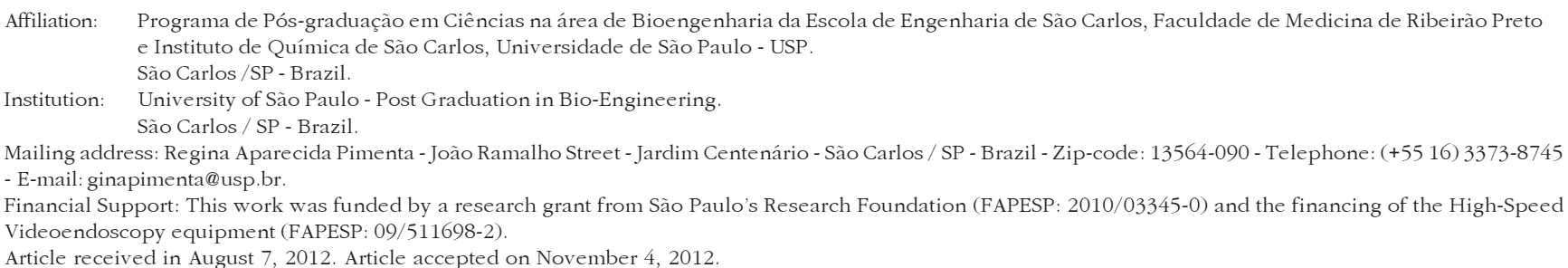

\section{SUMMARY}

Introduction: The effects of voiced vibration technique can be assessed by laryngeal imaging. Kymographic images derived from high-speed videoendoscopy allow actual visualization of vocal folds vibration.

Purpose: The aim of this study is to identify the immediate effects of the voiced vibration technique in healthy vocal folds using high-speed digital laryngeal imaging.

Methods: Samples were obtained from 15 healthy subjects with no history of voice disorders (6 men and 9 women aged 21 to 43 years). High-speed videoendoscopy recordings were performed before and after the voiced vibration technique. Kymographic images were obtained using high-speed videoendoscopy. The vocal folds were examined in their open and closed positions and the characteristics of the opening and closing phases were determined. A customize computational routine was used quantify these parameters. The closing, opening, and speed quotients were also calculated.

Results: In this study, women displayed statistically significant differences in opened phase $\left(\mathrm{P}=0.05^{*}\right)$, closed phase $\left(\mathrm{P}=0.046^{*}\right)$, and closing phase $\left(\mathrm{P}=0.026^{*}\right)$ phase characteristics. Men displayed the highest difference rate in opening time characteristics $(\mathrm{P}=0.06)$. The closing and opening quotients for the female group showed significant differences $\left(\mathrm{P}=0.029^{*}\right.$ and $\mathrm{P}=0.049^{*}$, respectively). The speed quotient exhibited statistically significant differences in the male group ( $\left.P=0.048^{*}\right)$.

Conclusion: The kymographic images indicated that the immediate effect of the voiced vibration technique was smooth contact in healthy vocal fold vibration.

Key words: High-speed digital imaging, Kymography, Voiced vibration, Voice.

\section{INTRODUCTION}

Semi-occluded vocal tract exercises are used in the treatment of dysphonia and in vocal warm-ups by professional voice users. These exercises are performed with the aim of reducing and minimizing the collision stress caused by vocal fold vibrations (1). The voiced vibration technique is one exercise that has been widely used by speech therapists empirically $(2,3)$. Voiced vibrations are performed with fast and repeated oscillations of the lips or tongue by rapid passage of the expiratory airflow along with phonatory emission (4). The voiced vibration technique is indicated for most vocal disorders, and has attracted the attention of researchers over the last few years (3).
According to the literature, a greater nonlinear interaction between the vocal tract and glottal source and increased supra- and subglottic pressures exert positive effects on voiced vibrations by reducing the stress of voice production (1). The effects of vocal exercises are assessed by modern instrumentation for voice analisys (2). Highspeed digital image-recording systems are the most current videoendoscopy methods used for voice studies. These systems allow reasearchers to observe the true vibrational patterns of the vocal folds $(5,6)$. High-speed kymography yields laryngeal images extracted from high-speed videoendoscopy, thereby allowing quantitative analyses of vocal-fold vibratory functions $(5,6,7)$.

Analysis of digital kymographic images is performed 
through juxtaposition of the laryngeal images within the frames. This method a requires selection of the cutting position of the vocal fold images with the largest opening amplitude and subsequent measurement of the time phases, the opening and closing phases, the initial and latter phases of the vibratory cycle, and the amplitude of the vocal folds $(5,6)$. This method is useful to investigate the effects of vocal exercises. The understunding of these effects is crucial in speech therapy; therefore, the goal of the present study was to identify the immediate effects of the voiced vibration exercise in healthy vocal folds by highspeed kymographic analysis.

\section{METHOD}

The study was approved by the Research Ethics Committee of the Federal University of São Paulo (UFSCar), protocol number 256/2010. All participants read and signed the Term of Free and Informed Consent, according to Resolution 196/96.

\section{Participants}

This was a prospective study conducted at the Voice Research Laboratory of the Hospital das Clínicas (HC-FMUSP) and School of Medicine, University of São Paulo. The inclusion criteria were the absence of medical reports for voice problems, no smoking and low alcohol consumption, no health problems during the voice testing, and normal perceptual quality of voice julged by a SLP voice specialist. Fifteen subjects ( 6 men and 9 women; ages ranging from 21 to 43 years) participated in this investigation.

\section{Equipment}

The data were extracted from high-speed videoendoscopy recordings before and after the voiced vibration exercises. Videoendoscopy data were acquired and stored by a system (Richard Wolf, Model HRES ENDOCAM 5562; Knittlingen, Germany) that acquires images at a rate of 4000 frames per second with a spatial resolution of 256x 256 pixels. A 90-degree rigid endoscope with a camera was used to record sequences of laryngeal images. The system is coupled to a computer and color video monitor (Figure 1).

The first high-speed videoendoscopy examination was performed before the vocal exercise. After the subject was seated in the examining chair, an endoscope was inserted into the subject's open mouth and advanced to the posterior pharynx. The participant was instructed

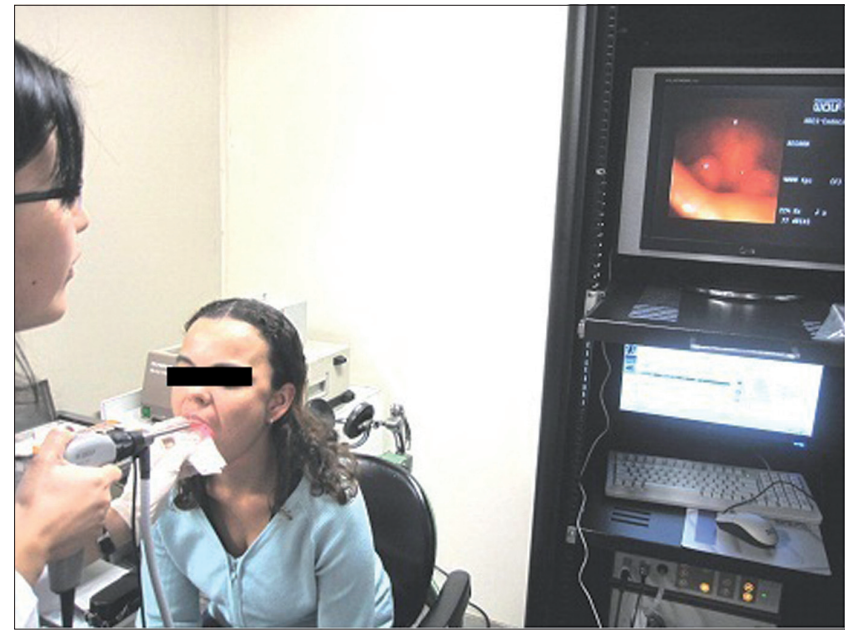

Figure 1. Laryngoscopic examination performed with the Richard Wolf equipment for high-speed image recordings. The laryngeal images are captured at a rate of 4000 frames per second. The equipment is composed of rigid endoscope (left) with camera (Endocam - 5562) coupled to a 300W light source (AUTO LP 5132) and a computer with color monitor (right) for image transmission and data analysis.

to produce the vowel /E/ while the tongue was held by the clinician (Figure 1). Sustained phonation of the /E/ vowel was produced at normal pitch and loudness. The endoscope was adjusted such that it was parallel to the superior surface of the vocal folds. The recording time for each videoendoscopy session was 2 seconds ( 4000 frames/s).

\section{Experimental Procedures}

The next step was to perform the vocal exercise. Each participant was instructed by a speech therapist to produce fast and repetitive oscillations of the lips or tongue together with voice emission. The voiced vibrations were produced at habitual pitch and loudness. The female subjects performed the vocal exercise for 3 minutes, and the male subjects for 5 minutes (11).

The second high-speed videoendoscopy examination was performed after the voiced vibration exercise. In this evaluation, the rigid endoscope was maintained in the larynx at the same angle and distance as that used in the vocal exercise. During the data-collection procedure, the fundamental frequency and emission intensity were controlled. The fundamental frequency was controlled by a virtual keyboard of the SpeechPitch software and intensity was measured by a decibel meter (RadioShack, Model 33-2055; New York, USA) placed $30 \mathrm{~cm}$ from the labial commissure of the subject. 


\section{Analysis of High-Speed Kymograph Parameters}

High-speed video recordings were converted into AVI files and processed by the software of the image recording system. The high-speed kymograph images were obtained by a line in the medial section of the laryngeal image for the capture and juxtaposition of the vocal fold images over time (Figure 2) (5). The midpoint was chosen due to its largervocal fold mobility characteristics $(12,13)$. We developed a customize computational routine to quantify, in milliseconds, the phases of the vibratory cycle of the vocal folds. We quantified the closed, open, closing and opening phases, as well as, the closing, opening, and speed quotients (Figure 3). The closed quotient is an estimation of the fraction of time that the vocal folds are closed during each vibratory cycle. The opened quotient is an estimation of the time that the vocal folds are open during each vibratory cycle, and speed quotient is a relationship between the opening and closing times of the vocal folds (14).

\section{Statistical analysis}

The results of the analyses before and after of the vocal exercise were compared using the paired $t$ test with a significance level of 0.05 .

\section{RESULTS}

The results of the statistical analysis are presented in Figures 4, 5, and 6, and in Tables 1 and 2. There were statistical differences in the following parameters for female vocal folds: opened phase, closed phase, closing phase, and closing and opening quotients. Male vocal folds showed statistical differences in the speed quotient. Results show that opened and closing phases increase for female ( $p=0.05$ and $p=0.026$, respectively). The closed phase decrease for female vocal folds $(\mathrm{p}=0.046)$. For female subjects the opening quotient increase $(\mathrm{p}=0.049)$ and decrease in the closing quotient $(p=0.029)$. Male vocal folds showed a decrease in the speed quotient $(p=0.048)$.

\section{DISCUSSION}

Voiced vibration is a semi-occluded vocal tract exercise that allows vocalization with normal intensity and less mechanical trauma to tissues during phonation. This exercise directs the vocal fold oscillation condition, in which the vocal folds are slightly adduced by retroflex pressure in the vocal tract. Glottal airflow and vocal fold collision are then minimized (1). In this study, the goal was
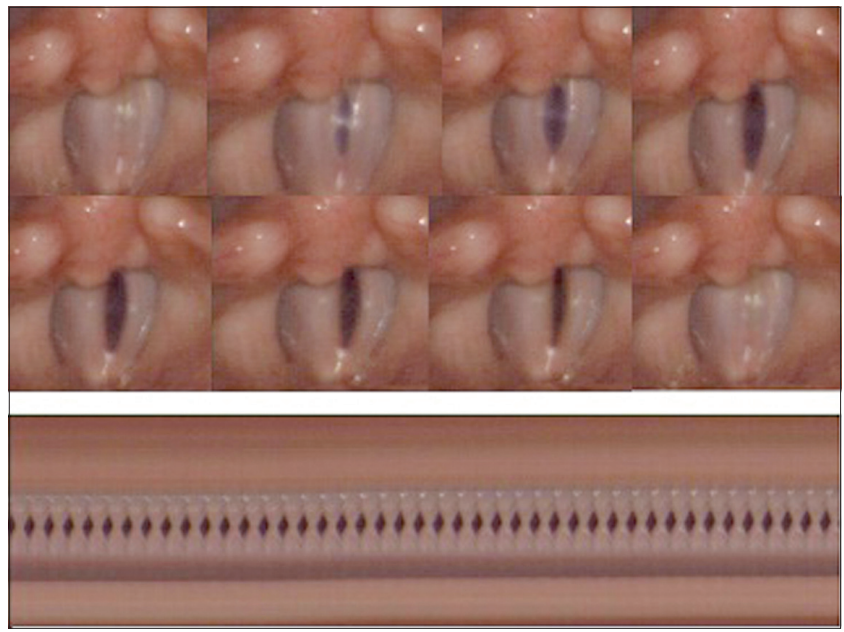

Figure 2. Top: Clipping of one vibration period from a highspeed recorded sequence of male healthy vocal folds. Bottom: High-speed kymography from high-speed recording of the sequence displayed at the top.

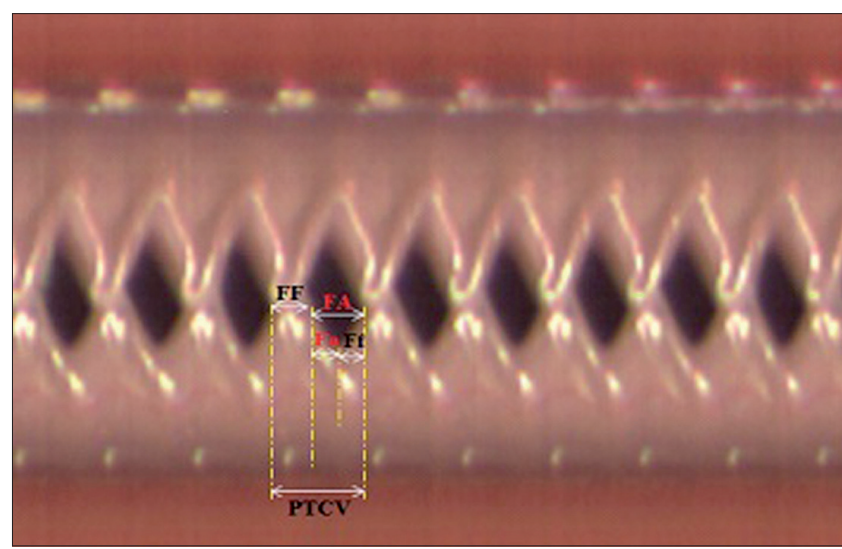

Figure 3. Kymographic image showing vibratory cycle phases. PTCV: total phase of a single vibratory cycle, FF: closed phase, FA: opened phase, Ff: closing phase, and Fa: opening phase.

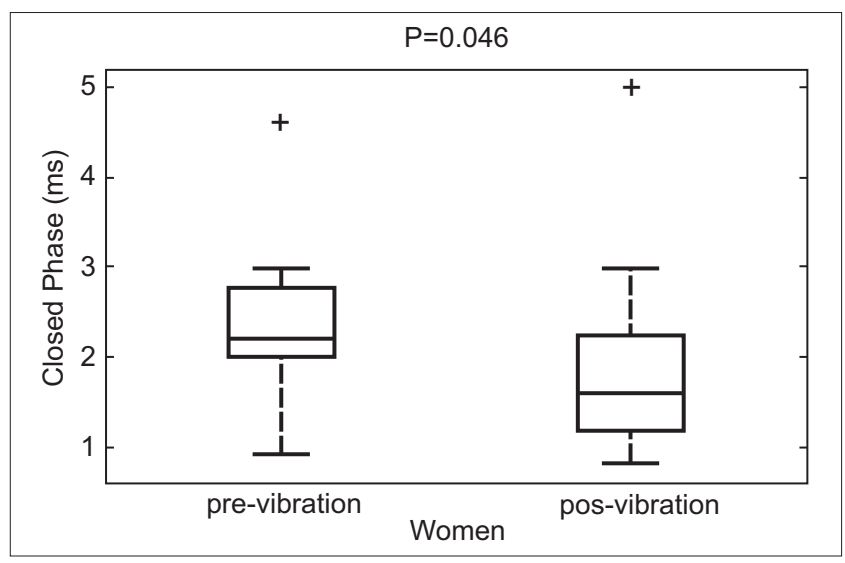

Figure 4. Distribution of average values and standard deviation of the closed phase extracted before and after vibration exercise by high-speed kymography of female vocal folds. 


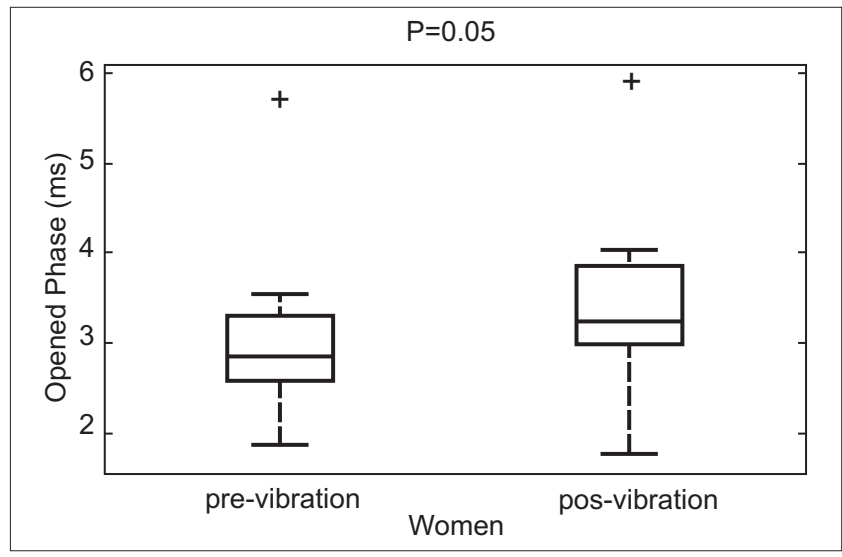

Figure 5. Distribution of average values and standard deviation of the opened phase extracted before and after the vibration exercises by high-speed kymography of female vocal folds.

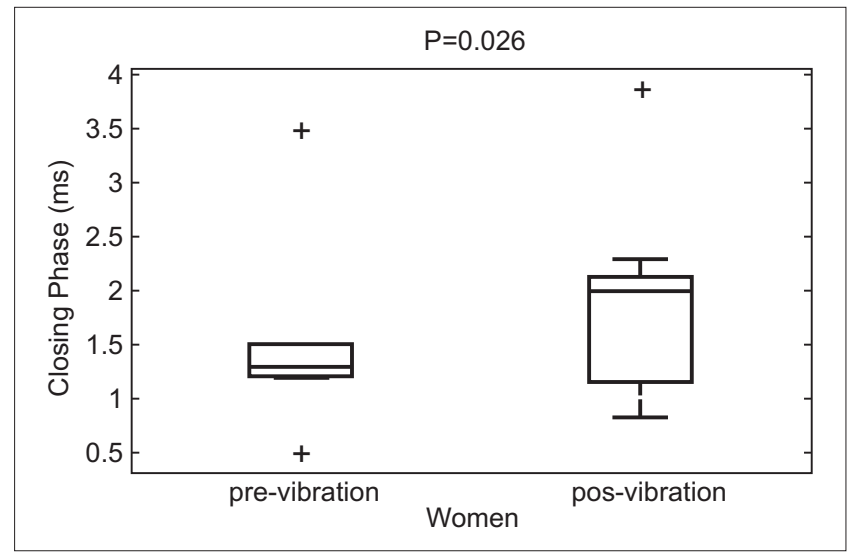

Figure 6. Distribution of average values and standard deviation of the closing phase extracted before and after the vibration exercises by high-speed kymography of female vocal folds.

Table I. Comparison between quotients before and after the vibration exercise offemalevocal folds. Mean (M), Standard Deviation(SD), minimum and maximum values and significance $(P)$ of opened quotient $(O Q)$, closed quotient $(C Q)$, and speed quotient(SQ).

\begin{tabular}{lcccccc}
\hline Parameters & \multicolumn{2}{c}{ OQ } & \multicolumn{2}{c}{ CQ } & \multicolumn{2}{c}{ SQ } \\
& before & after & before & after & before & after \\
\hline M & 0.56 & 0.63 & 0.44 & 0.35 & 1.25 & 1.08 \\
SD & 0.11 & 0.13 & 0.11 & 0.15 & 0.56 & 0.66 \\
Minimum & 0.38 & 0.36 & 0.21 & 0.18 & 0.63 & 0.60 \\
Maximum & 0.79 & 0.82 & 0.62 & 0.64 & 2.66 & 2.63 \\
P & $0.049 *$ & $0.029 *$ & \multicolumn{2}{c}{0.57} \\
\hline
\end{tabular}

Paired ttest with significance level 0.05 .

Table 2. Comparison between quotients before and after the vibration exercise of malevocal folds. Mean (M), Standard Deviation (SD) minimum, maximum and Significance $(P)$ of opened quotient $(\mathrm{OQ})$; closed quotient $(\mathrm{CQ})$; speed quotient (SQ).

\begin{tabular}{lcccccr}
\hline Parameters & \multicolumn{2}{c}{ OQ } & \multicolumn{2}{c}{ CQ } & \multicolumn{2}{c}{ SQ } \\
& before & after & before & after & before & after \\
\hline M & 0.53 & 0.55 & 0.40 & 0.37 & 1.48 & 1.31 \\
SD & 0.19 & 0.20 & 0.15 & 0.15 & 0.91 & 1.18 \\
Minimum & 0.46 & 0.47 & 0.35 & 0.34 & 1.00 & 0.54 \\
Maximum & 0.79 & 0.82 & 0.62 & 0.64 & 3.80 & 4.52 \\
P & \multicolumn{2}{c}{0.15} & \multicolumn{2}{c}{0.10} & \multicolumn{2}{c}{$0.048 *$} \\
\hline
\end{tabular}

Pairedttest with significance level0.05.

to identify the immediate effects of voiced vibrations in healthy vocal folds. Thus, we applied a high-speed kymograph analysis method. Our results corroborated the effect described by Titez (1). The vibratory patterns of the female vocal folds showed statistically significant differences. The results indicate soft contact between the female vocal folds due to decreased contact speed of the mucosa $(\mathrm{P}=$ 0.026* for the closing phase), and the time the vocal folds remained closed ( $\mathrm{P}=0.046^{*}$ for the closed phase). Furthermore, there was an increase in the time the vocal folds remained open $(\mathrm{P}=0.05 *$ for the opened phase $)$. The changes in the vibratory patterns of the male group suggest soft contact between vocal folds during phonation. The results point to a slow contact of the vocal fold mucosa ( $\mathrm{P}=0.08$ for the closing phase), larger opening speed ( $\mathrm{P}$ $=0.06$ in the opening phase), and an increase in the time the glottis remained in the open position ( $\mathrm{P}=0.06$ for the opened phase). However, there were no significant results for the males, indicating the need for further research with a larger group of subjects. 
This is the first study of the effects of vocal techniques through kymographic parameters. Interestingly, our results agree with those of a videostroboscopic analysis (15). However, Pereira et al. study investigated female voices after applying a set of vocal techniques that included voiced vibrations. GASKILL and ERICKSON studied voiced vibrations by estimating the closing quotient on the basis of electroglottographic signals (EGG). The authors found a lower closing quotient during the voiced vibrations, concluding that for the glottic cycle to occur during voiced vibrations, the airflow has to be increased to sustain the vocal fold and lip oscillation (16). In our study, the closed quotient was extracted from the high-speed kymographs, and these results are in agreement with the conclusions presented by Gaskill and Erickson (16). The results can be interpreted to represent the changes in the vibratory pattern behavior of the vocal folds with reduced mechanical trauma of the tissues during the vocal exercise.

The pre- and post-exercise data for the quotients provided more support than the kymographic parameter data. In the female vocal folds, the effect produced by vocal exercise is evidenced by the decreased closing quotients ( $\left.\mathrm{P}=0.029^{*}\right)$, indicating the less time needed for the contact between vocal folds than for the entire glottic cycle. Furthermore, the average opening quotient increased $(\mathrm{P}=0.049 *)$, indicating an increase in the time the glottis remained in the open position throughout the glottic cycle. These relationships showed the expected voiced vibration effect; in other words, after the vocal exercise, the glottis remains open longer. In the male vocal folds, the expected effect of the exercise was indicated by the speed quotient ( $\mathrm{P}=0.048^{*}$ ), which showed a significant decrease in its average value after the voiced vibrations. This could be due to the increase in the speed of mucosal contact in the vocal folds. This behavior can be understood as an effect that prioritizes the slow closing and fast contact of the vocal folds to avoid the mechanical trauma collisions during phonation.

\section{CONCLUSIONS}

This study allowed identification of the immediate effect of soft contact between the healthy vocal folds after the voiced vibration. The female vocal folds exhibited greater susceptibility to the influence of the vocal exercise. However, further research with a greater number of samples, mainly male samples, is necessary to corroborate the results of this paper.

High-speed kymography proved to be an efficient voice evaluation tool that could be used in other vocal exercise studies.

\section{ACKNOWLEDGMENTS}

To the Bio-Engineering Post graduate program University of São Paulo-São Carlos. To the members of the Otolaryngology Department, School of Medicine, University of SãoPauloand to the BiomedicalSignal ProcessingLaboratory of EESC/USP. This work was funded by a research grant from São Paulo's Research Foundation (FAPESP:2010/03345-0) and the financing of the High-Speed Videoendoscopy equipment (FAPESP: 09/511698-2).

\section{REFERENCES}

1. Titze IR. Voice Training and therapy with a semi-occluded vocal tract: rational and scientific underpinnings. J Speech Lang Hear Res. 2006;49:448-59.

2. Elliot N, Sundberg J, Gramming P. Physiological aspects of a vocal exercise. J Voice. 1997;11(2):171-7.

3. Azevedo LL, Passaglio KT, Rosseti MB, Silva CB, Oliveira BF, Costa RC. Avaliação da performance vocal antes e após a vibração sonorizada de língua. Rev Soc Bras Fonoaudiol. 2010;15(3):343-8.

4. Schwarz K, Cielo CA. Modificações laríngeas e vocais produzidas pela técnica de vibração sonorizada de língua. Pró-Fono. 2009;21(2):161-6.

5. Tsuji DH, Sennes LU. Videoquimografia de laringe: novo método de avaliação da vibração cordal. Arq Fund Otorrinolaringol. 1998;2(4):136-40.

6. Koishi HU, Tsuji DH, Imamura R, Sennes LU. Variação da intensidade vocal: estudo da vibração das pregas vocais em seres humanos com videoquimografia. Rev Bras Otorrinolaringol. 2003;69(4):464-70.

7. Yan Y; Damrose E; Bless D. Functional Analysis Of Voice Using Simultaneous High-Speed Imaging and Acoustic Recordings. J Voice. 2007;21(5):604-16.

8. Gasparini G, Behlau M. Quality of life: validation of the Brazilian version of the voice-related quality of life (V-RQOL) measure. J Voice. 2009;23(1):76-81.

9. Behlau M, Oliveira G, Santos La, Ricarte A. Validação no Brasil de protocolos de auto-avaliação do impacto de uma disfonia. Pró-Fono. 2009;21(4):326-32.

10. Yamazaki R, LeãoSHS, Madazio G, Padovani M, Azevedo $\mathrm{R}$, Behlau M. Correspondência entre escala analógico-visual e a escala numérica na avaliação perceptivo-auditiva das 
vozes. XVI Congresso Brasileiro de Fonoaudiologia, 2008, Campos do Jordão (SP).

11. Menezes MH, de Campos Duprat A, Costa HO. Vocal and laryngeal effects of voiced tongue vibration technique according to performance time. J Voice. 2005;19(1):61-70.

12. Kurita, S. Layer structure of the human vocal fold: morphological investigation. Otologia (Fukuoka). 1980;26:973-97.

13. Haji T, Isshiki N, Mori K, Omori K, Taira T, Honjo I. Experimental study of the mobility of the vocal fold mucosa. Folia Phoniatr. 1991;43(1):21-8.
14. Hirano M. Phonosurgery. Basic and clinical investigations. Otologia (Fukuoka) (Suppl 1) 1975;21:239-440.

15. Pereira EC, Silvério KCA, Marques JM, Camargo PAM. Efeito imediato de técnicas vocais em mulheres sem queixa vocal. Rev CEFAC. 2011;13(5):886-94.

16. Gaskill CS, Erickson ML. The effect of a voiced lip trill on estimated glottal closed quotient. Journal of Voice. 2008;22(6):634-43. 